Rolf Arnold

Führen mit Gefühl 
Rolf Arnold

\section{Führen mit Gefühl}

Eine Anleitung zum Selbstcoaching

Mit einem Methoden-ABC

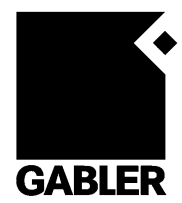


Bibliografische Information Der Deutschen Nationalbibliothek

Die Deutsche Nationalbibliothek verzeichnet diese Publikation in der

Deutschen Nationalbibliografie; detaillierte bibliografische Daten sind im Internet über

$<$ http://dnb.d-nb.de> abrufbar.

\section{Auflage 2008}

Alle Rechte vorbehalten

(C) Betriebswirtschaftlicher Verlag Dr. Th. Gabler I GWV Fachverlage GmbH, Wiesbaden 2008

Lektorat: Ulrike M. Vetter I Stefanie A. Winter

Der Gabler Verlag ist ein Unternehmen von Springer Science+Business Media.

www.gabler.de

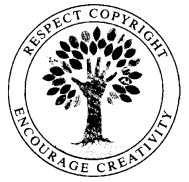

Das Werk einschließlich aller seiner Teile ist urheberrechtlich geschützt. Jede Verwertung außerhalb der engen Grenzen des Urheberrechtsgesetzes ist ohne Zustimmung des Verlags unzulässig und strafbar. Das gilt insbesondere für Vervielfältigungen, Übersetzungen, Mikroverfilmungen und die Einspeicherung und Verarbeitung in elektronischen Systemen.

Die Wiedergabe von Gebrauchsnamen, Handelsnamen, Warenbezeichnungen usw. in diesem Werk berechtigt auch ohne besondere Kennzeichnung nicht zu der Annahme, dass solche Namen im Sinne der Warenzeichen- und Markenschutz-Gesetzgebung als frei zu betrachten wären und daher von jedermann benutzt werden dürften.

Umschlaggestaltung: Nina Faber de.sign, Wiesbaden

Druck und buchbinderische Verarbeitung: Wilhelm \& Adam, Heusenstamm

Gedruckt auf säurefreiem und chlorfrei gebleichtem Papier

Printed in Germany

ISBN 978-3-8349-0791-2 


\title{
Vorwort
}

\author{
„Wenn du veränderst, \\ verändert sich nichts. \\ Denn jede Veränderung muss \\ Selbstveränderung sein.“
}

(Willke 1987, S. 350)

\begin{abstract}
Dieses Buch beinhaltet einen Briefwechsel zwischen einer Führungskraft und ihrem ehemaligen Mentor, der sie in schwierigen Phasen begleitet hat. Diese Begleitung findet in diesen Briefen ihre Fortsetzung. Dabei wird ein Coachingprozess sichtbar, der tief an der Person und ihren Gefühlen, Fragen und Zweifeln ansetzt, gleichzeitig jedoch auch unmittelbare Ratschläge anbietet, wie sie uns die Veränderungswissenschaften nahelegen. Es wird deutlich, dass alle Fragen, Probleme und Zwangslagen, in denen wir uns befinden, auch Ausdrucksformen des Mechanismus sind, dass im Außen nur sein darf, was auch im Innen sein darf. Dies ist die Strukturdeterminiertheit unserer Wahrnehmung, von welcher der Konstruktivismus spricht.
\end{abstract}

Wir können uns die Welt nur zu unseren eigenen Bedingungen konstruieren, und wir tun dies so, wie wir es aushalten können. In dem, was wir erleben, begegnet uns deshalb oft mehr Eigenes als wirklich Neues. Die Beleuchtungen, Handlungsimpulse und Gefühle, die sich von diesem vertrauten Eigenen her beständig in unser Leben einmischen, zu erkennen, ist ein wesentliches Ziel eines emotionalen Coachings, wie es aus dem hier vorgelegten Briefwechsel spricht. Dabei wird auch deutlich, dass die Fragen, mit denen man sich in der Krisenbegleitung konfrontiert sieht, teilweise uneinholbar sind. Nicht alle Fragen finden eine Antwort, oftmals ist das Einzige, was gelernt werden kann, das richtige Fragen. Die Erschütterungen, die eine Krise im Leben einer Führungskraft auszulösen vermag, können weite Kreise ziehen, denn Führungskrisen sind zumeist Persönlichkeitskrisen, und Krisen im persönlichen Leben wirken sich auch im kooperativen Handeln der Menschen aus. Aus diesem Grunde muss Coaching ganzheitlich orientiert sein, Beratung ist nicht auf einzelne Segmente der Führungskompetenz zu begrenzen. 
Menschen haben oftmals keinen Zugang zu ihren Emotionen. Dies gilt auch für Führungskräfte, und es gilt für sie in einem besonderen Maße. Es scheint so, als sei eine gewisse Distanz zu der eigenen inneren Substanz häufig eine Art Voraussetzung dafür, nach Führung zu streben. Dann wird Führung uneigentlich, da ihre Entscheidungen und Handlungen sich nach Maßgaben bemessen, die nicht der konkreten Lage, sondern eigenen Resonanzanliegen geschuldet sind. Solche Führungskräfte spüren sich in ihrem Führungshandeln, weshalb sie oftmals nicht spüren, was für die Situation, die Organisation und die in dieser Tätigen das Notwendige und Erforderliche ist. Die emotionale Kompetenz und die Wirksamkeit von Führungskräften sind deshalb wesentliche Voraussetzungen für den systemischen Erfolg ihres Handelns. Systemisch ist ein Erfolg, der die Potenziale und Ressourcen einer Organisation optimal bündeln und zur Entfaltung zu bringen vermag.

Dies gelingt Führungskräften in der Regel nur, wenn sie z. B. in einem Coaching gelernt haben, sich von außen zu beobachten und die eigentlichen Triebkräfte ihres Handelns zu verstehen. Gute Führungskräfte müssen zuallererst die Führung in ihrem eigenen Leben übernehmen und „Herren im eigenen Haus“ ihrer Seele und Motive werden. Nur, wer eine Vision von gelungenem Leben in seinem Herzen trägt, kann auch wirklich führen. Denn das Wohin der Führung liegt stets außerhalb der eigenen unbewussten Anliegen und zumeist auch außerhalb des eigenen begrenzten Zeithorizontes. Führungskräfte müssen achtsam und auch unerschrocken sein. Ihr Führungsanspruch ergibt sich aus einer abschiedlichen Grundeinstellung zum Leben einerseits und einer bewussten Entscheidung zu den verbleibenden Möglichkeiten dieses Lebens andererseits. Emotionale Kompetenz kann deshalb auch nicht in einem Schnellkurs erworben werden, sie ist stets das Resultat einer persönlichen Transformation, d. h. eines Aufbruchs zu einer selbstreflexiven Beobachterhaltung, einer neuen biographischen Gewissheit und einer systemischen Bescheidenheit. Wer sein eigenes Erleben für real, seine Sicht der Dinge für objektiv richtig und die Erfüllung seiner tief verwurzelten Geltungssüchte für sachlich erforderlich hält, der mutet sich den anderen einfach nur so zu, wie er seine Welt fühlt und versteht. Er ist mit dieser Haltung zwar einige Zeit zu ertragen und vielleicht auch tüchtig, wird aber nur selten in der Lage sein, die Potenziale und Ressourcen der Menschen, für die er Verantwortung trägt, wirklich zur Entfaltung zu bringen. Dies merken Organisationen spätestens dann, wenn die Kreativität und Selbststeuerung für sie zu entscheidenden Wettbewerbsfaktoren werden. Dann zeigt sich die Begrenztheit von Organisationsformen, die auf die narzisstischen Anliegen Einzelner zugeschnitten sind und nach Maßgabe ihrer Bedürftigkeiten funktionieren sollen. Solche Organisationsformen sind noch immer in einem Dornröschenschlaf gelähmt, und es bedarf nicht des mutigen Königssohnes, um sie aufzuwecken, sondern der Führungskraft, deren Mut darin besteht, sich zu sich selbst aufzumachen. 
Dieses Buch zeichnet diesen Aufbruch einer Führungskraft zu sich selbst detailliert in einem Briefwechsel zwischen einem Coach und seinem Klienten nach. Gleichwohl ist dieses Buch ein Artefakt. Es gibt weder Karl noch Bernhard als Personen. Es gibt jedoch die Gefühle, Fragen und Zweifel von Führungskräften, wie ich aus zahlreichen eigenen Forschungs- und Beratungskontakten der letzten Jahre sowie aus eigener Leitungsfunktion in unterschiedlichen Organisationen weiß. Die in dieser Arbeit gesammelten Erfahrungen sind in den hier vorgelegten Briefwechsels eingeflossen.

Februar 2008

Rolf Arnold 


\section{Inhaltsverzeichnis}

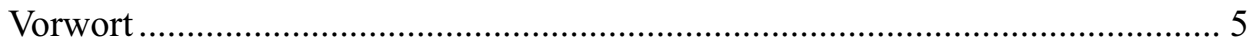

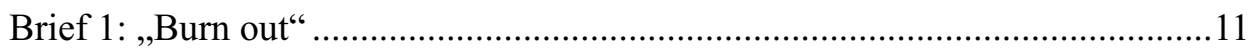

Brief 2: Selbstarchäologie als Ich-Arbeit .................................................... 15

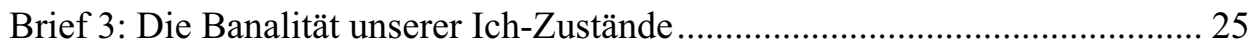

Brief 4: „Ich übernehme die Verantwortung!“................................................. 37

Brief 5: „Ich experimentiere mit meinen Beobachtungsgewohnheiten!“........... 53

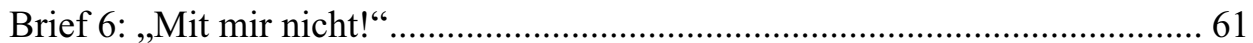

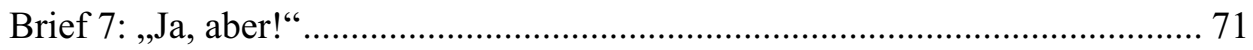

Brief 8: Die Suche nach dem persönlichen Referenzpunkt ................................ 83

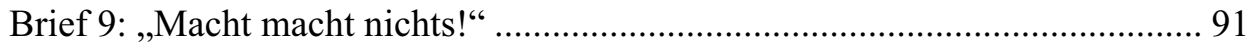

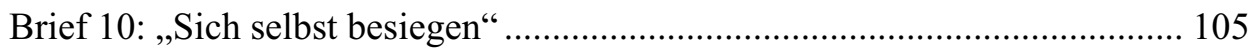

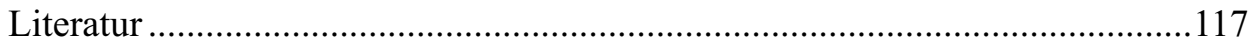

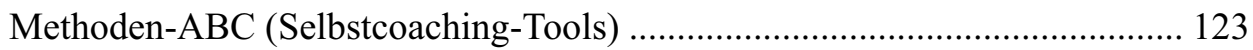

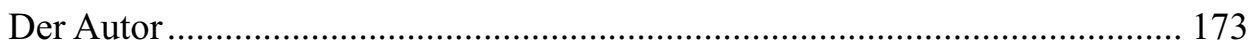

